\title{
Avaliação da Maturidade Pulmonar Fetal em Gestaç̧ess de Alto Risco
}

\author{
Prenatal Diagnosis of Fetal Lung Maturity in High-Risk Pregnancies
}

Wladimir Taborda ${ }^{1}$, Maria Fernanda B. de Almeida ${ }^{2}$

Antonio F. Moron ${ }^{1}$, Anna Maria Bertini ${ }^{1}$

\begin{abstract}
RESUM0
Trata-se de um estudo prospectivo para a avaliação da maturidade fetal em 121 gestações de alto risco realizado no Hospital São Paulo - Universidade Federal de São Paulo, entre janeiro de 1990 e janeiro de 1995. Em todos os casos, o parto foi realizado em até 3 dias após a obtenção de líquido amniótico por amniocentese. O objetivo principal foi o de analisar a acurácia do teste de Clements (TC), da relação lecitina/esfingomielina (L/E), da presença de fosfatidilglicerol (PG) e do perfil pulmonar (relação $L / E>1,7$ e PG presente) para antecipar a ocorrência ou não de sindrome de desconforto respiratório neonatal (SDR). Foram calculados a sensibilidade, a especificidade e os valores preditivos positivo (VPP) e negativo (VPN) de todos os testes. O grupo de estudo foi composto por 48 gestações complicadas por diabetes mellitus, 41 por síndromes hipertensivas, 14 por isoimunização Rh e 18 por diversas patologias. O perfil pulmonar apresentou sensibilidade de $100 \%$ em todos os casos. O teste de Clements também não apresentou resultados falso-positivos em gestantes hipertensas, apurando-se, contudo, de 20\% a 50\% de falso-negativos em todos os outros testes. Os quatro testes apresentaram baixos VPP (23\% no TC, 51\% na relação L/E, 63\% na presença de PG, 61\% no perfil pulmonar) e elevados VPN (92\% no TC, 88\% na relação L/E, 89\% na presença de $P G, 100 \%$ no perfil pulmonar). Este estudo demonstrou que a presença de PG $e$ relação $L / E>1,7$ simultâneos no líquido amniótico comprovam a maturidade pulmonar com muito baixo risco de DR ao nascimento. Concluiu-se também que o teste de Clements deve constituir o rastreamento inicial para predizer a ausência de SDR, particularmente em gestações complicadas por síndromes hipertensivas.
\end{abstract}

PALAVRAS-CHAVE: Maturidade pulmonar fetal. Amniocentese. Complicações da gestação. Diabetes mellitus. Hipertensão arterial. Teste de Clements. Relação L/E.

${ }^{1}$ Disciplina de Obstetrícia

${ }^{2}$ Disciplina de Pediatria Neonatal

Escola Paulista de Medicina

Universidade Federal de São Paulo - SP

Correspondência:

Wladimir Taborda

Rua Dr. Alceu de Campos Rodrigues, 247/53, Itaim

CEP 04544-000, São Paulo/SP

Fone/Fax 0118219370

\section{Introdução}

O parto pré-termo é a principal causa de mortalidade perinatal em todo o mundo. Com incidência estimada de $10 \%$ em nosso meio, apresenta etiologia multifatorial ${ }^{6}$. Condições socioeconômicas desfavoráveis, precária 
assistência pré-natal e presença de infecções genitourinárias são os principais fatores predisponentes para a prematuridade "espontânea", geralmente secundária ao trabalho de parto prematuro e à rotura prematura das membranas, patologias de alta prevalência e difícil prevenção ${ }^{17}$.

A sindrome de desconforto respiratório (SDR) persiste como a principal causa primária de mortalidade neonatal em conceptos pré-termo, sendo conseqüente à imaturidade alveolar e deficiente sintese de surfactante pulmonar. Embora o advento da ventilação mecânica tenha melhorado o prognóstico imediato de recém-nascidos que apresentam SDR, muitos efeitos adversos do tratamento têm sido relatados. As complicações mais comuns decorrentes da ventilação mecânica incluem a displasia broncopulmonar, sepse, estenose subglótica e enfisema pulmonar ${ }^{14}$.

Por outro lado, a antecipação oportuna do parto pode representar uma intervenção terapêutica valiosa, e até mesmo salvadora, em diversas gestações de risco. Síndromes hipertensivas, diabetes mellitus, isoimunização pelo fator $\mathrm{Rh}$, insuficiência placentária ou crescimento fetal retardado são exemplos comuns, nos quais os riscos e benefícios maternos e perinatais do parto prétermo eletivo devem ser criteriosamente avaliados.

Neste contexto, a determinação da maturidade pulmonar fetal representa um importante elemento para a conduta obstétrica, tendo-se em mente que evitar o nascimento prematuro constitui a principal estratégia preventiva para reduzir os riscos e seqüelas da morbidade respiratória neonatal. A determinação acurada da maturidade pulmonar fetal, todavia, tem sido um problema crucial em perinatologia. Praticamente todos os métodos propostos por diferentes pesquisadores têm como objetivo avaliar o risco de ocorrência de SDR. Os métodos diretos, e geralmente mais eficazes, são aqueles que utilizam amostras de líquido amniótico para análise específica dos componentes do surfactante pulmonar.

A medida da relação lecitina/esfingomielina $(\mathrm{L} / \mathrm{E})$ através de cromatografia em camada fina é uma das provas laboratoriais mais comumente utilizadas para avaliar a maturidade pulmonar fetal. Diversos estudos comprovaram que a técnica é segura na identificação de conceptos com pulmões imaturos e de risco elevado para $\mathrm{SDR}^{4,9,10}$. A identificação de fosfatidilglicerol (PG) no líquido amniótico também parece oferecer garantia de maturidade pulmonar ao nascimento ${ }^{12}$. Ademais, o PG não foi detectado no sangue, mecônio ou secreções vaginais (contaminantes comuns do líquido amniótico) e, portanto, não há confusão na interpretação da maturidade pulmonar.
A cromatografia em placa fina sobre gel de sílica permite uma separação mais completa dos fosfolipídios surfactantes no líquido amniótico ${ }^{13}$. As porcentagens de fosfatidilcolina dessaturada, fosfatidilglicerol e fosfatidilinositol (PI) podem ser analisadas simultânea e satisfatoriamente por meio desta técnica, mesmo em amostras contaminadas por sangue ou mecônio. Assim, a maturidade pulmonar é expressa não só pela relação L/E, como também pela associação com outros fosfolipídios importantes ${ }^{11}$. O uso deste perfil pulmonar aumenta muito a exatidão da técnica e virtualmente elimina os resultados intermediários (e nãoinformativos), sendo especialmente útil no tratamento de gestações de alto risco.

O teste de Clements, "shake test", teste da espuma ou teste rápido de surfactante é o método mais amplamente utilizado na prática obstétrica diária para determinação da maturidade do pulmão fetal ${ }^{5}$. O objetivo primordial consiste em reduzir o tempo e a complexidade inerentes à medida exata da concentração de fosfolípides por meio de técnicas cromatográficas. É o protótipo do teste rápido, barato e simples e baseia-se na propriedade do surfactante em formar bolhas de espuma estáveis na interface ar-líquido na presença de etanol.

Os falsos resultados de qualquer teste podem ser conseqüência da presença de patologias maternas que determinam anormalidades metabólicas ou de perfusão uteroplacentária, associadas ao retardamento ou à aceleração da maturação pulmonar fetal. O intervalo de tempo entre a obtenção do líquido amniótico e o parto também afetam criticamente a acurácia de qualquer teste. O valor preditivo positivo ("imaturo") diminui com o aumento do intervalo, sendo que a maioria dos estudos inclui somente casos com intervalo máximo de 72 horas entre a amniocentese e o parto $8,10,16,19$.

O propósito do presente estudo foi verificar a sensibilidade, a especificidade e os valores preditivos positivo e negativo do teste de Clements, da relação lecitina/esfingomielina, da presença de fosfatidilglicerol e do perfil pulmonar em relação à ocorrência de SDR em gestantes de alto risco internadas no Hospital São Paulo (Disciplina de Obstetrícia da Universidade Federal de São Paulo) em um período de cinco anos.

\section{Pacientes e Métodos}

A população de estudo foi constituída por 121 gestantes de alto risco internadas no Hospital São Paulo - Disciplina de Obstetrícia da Universidade Federal de São Paulo (UNIFESP), que foram submetidas à amniocentese para avaliação pré-natal 
da maturidade pulmonar fetal no período compreendido entre janeiro de 1990 e janeiro de 1995.

Os critérios de inclusão constaram da obtenção de líquido amniótico não-contaminado por sangue e/ou mecônio e intervalo entre a amniocentese e o parto inferior a 72 horas. Os critérios de exclusão foram a natimortalidade, gestação gemelar, ministração materna de corticosteróides, índice de APGAR (1953) inferior a 7 no $5^{\circ}$ minuto de vida pós-natal e a rotura prematura de membranas.

A amniocentese foi realizada sob visão ecográfica direta de modo a evitar acidentes de punção. Foram obtidos de 7 a $10 \mathrm{ml}$ de líquido amniótico em cada punção para a realização simultânea do teste de Clements $(3 \mathrm{ml})$ e da cromatografia em camada fina unidimensional (4 ml). Nas 24 horas seguintes, a paciente foi rigorosamente observada em relação ao surgimento de dinâmica uterina anormal, sangramento vaginal ou amniorrexe prematura.

O líquido amniótico obtido foi utilizado para a realização simultânea do teste de Clements, relação L/E, pesquisa PG e perfil pulmonar. O perfil pulmonar neste estudo foi definido por relação L/E superior ou igual a 1,7 e presença simultânea de PG com o uso da cromatografia em camada fina unidimensional.

O teste de Clements ${ }^{5}$ foi realizado com três diluições diferentes de líquido amniótico $\left(1^{\circ}\right.$ tubo: $1.0 \mathrm{ml} ; 2^{\circ}$ tubo: $0.75 \mathrm{ml}$ e $0.25 \mathrm{ml}$ de solução fisiológica (SF) a 0,9\% e $3^{\circ}$ tubo: $0.50 \mathrm{ml}$ e $0.50 \mathrm{ml}$ de $\mathrm{SF}$ ) às quais foi adicionado $1.0 \mathrm{ml}$ de álcool absoluto em tubos de vidro de $14 \times 100 \mathrm{~mm}$, quimicamente limpos e previamente numerados. Os tubos, fechados com tampa de borracha limpa, foram vigorosamente agitados durante 15 segundos e colocados verticalmente em suporte adequado. A persistência de um anel intacto de bolhas na interface ar/líquido após 15 minutos de observação configurou o resultado positivo. Neste estudo, o teste foi dividido em duas categorias de resultados: (1) negativo ou "imaturo" - não-persistência de bolhas no $1^{\circ}$ tubo ou somente nos 2 primeiros tubos e ausente no $3^{\circ}$ e (2) positivo ou "maduro" - espuma estável até o $3^{\circ}$ tubo.

$\mathrm{Na}$ técnica para cromatografia em camada fina unidimensional o espécime de líquido amniótico foi centrifugado em velocidade lenta (1.000 rotações por minuto [rpm]) durante 3 minutos, sendo o sobrenadante removido imediatamente após a coleta. A quantificação cromatográfica utilizou o método Helena Fetal-Tek 200 (Helena Laboratories, Beaumont, TX, EUA), em que os fosfolipídios são extraídos da amostra de líquido amniótico através da mistura de clorofórmio-metanol, dispostos em uma placa de cromatografia de camada fina e separados, utilizando-se um sistema solvente que contém clorofórmio, metanol, 2-propanol, trietilamina e água destilada.

De acordo com a recomendação do fabricante, o laboratório da Disciplina de Obstetrícia da UNIFESP estabeleceu o seu próprio padrão de normalidade para a relação $\mathrm{L} / \mathrm{E}$, utilizando os marcadores padronizados que acompanham as placas de cromatografia, sendo a relação igual ou superior a 1,7 considerada o padrão de maturidade pulmonar bioquímica ${ }^{2}$. Desta forma, o perfil pulmonar foi considerado completo quando a relação $\mathrm{L} / \mathrm{E}$ foi igual ou superior a 1,7 e se constatou a presença de fosfatidilglicerol na mesma amostra $^{13}$.

A síndrome de desconforto respiratório (SDR) foi definida pela necessidade de ministração de oxigênio após o nascimento. A ventilação mecânica foi indicada sempre que a $\mathrm{pO}_{2}$ foi inferior a 50 $\mathrm{mmHg}, \mathrm{pCO}_{2}$ acima de $50 \mathrm{mmHg}$ ou aumento maior que $10 \mathrm{mmHg}$ por hora, e na vigência de apnéias recorrentes e acidose grave $(\mathrm{pH}<7,20)$.

O desempenho dos testes diagnósticos de maturidade pulmonar fetal foi avaliado pela comparação dos resultados destes ante a presença ou ausência de SDR no berçário. Foram calculadas a sensibilidade, a especificidade e os valores preditivos positivo e negativo de cada teste. A sensibilidade foi definida como a proporção de indivíduos com a doença (neonatos que apresentaram SDR) que tiveram um teste positivo para a mesma (resultado "imaturo"). A especificidade foi a proporção de indivíduos sem a doença (conceptos que não desenvolveram SDR) que tiveram um teste negativo para a mesma (resultado "maduro"). O valor preditivo positivo estimou a probabilidade de ocorrência da doença (SDR) nos casos em que o teste foi positivo (resultado "imaturo" - negativo do teste de Clements; relação L/E < 1.7; ausência de PG e ausência de perfil pulmonar completo) e o valor preditivo negativo estimou a probabilidade da ausência de SDR nos casos em que o teste foi negativo (resultado "maduro" - teste de Clements positivo; relação $\mathrm{L} / \mathrm{E}>1,7$; presença de fosfatidilglicerol e presença de perfil pulmonar completo). Para a relação L/E, em que o resultado foi expresso em uma escala intervalar contínua, foi possível estabelecer muitos pontos de corte e, tendo por base cada um deles, calcular suas respectivas sensibilidade e especificidade. Para tanto foi utilizada a chamada curva "ROC" ("receiver operator characteristic"). Esta curva foi construída em diagrama cartesiano em que cada ponto representa simultaneamente a sensibilidade e a especificidade de um determinado ponto de corte da relação $\mathrm{L} / \mathrm{E}$. 
A tabulação dos dados e a análise estatística para o presente estudo foram realizadas utilizandose o programa de computador de domínio público denominado Epi-Info, versão 5.01, de julho de 1991 ("Public Domain Software for Epidemiology and Disease Surveillance”), concebido por Jeffrey Dean, Andrew Dean, Anthony Burton e Richard Dicker no Centro de Controle de Doenças, Atlanta, GA, EUA e divulgado pela Organização Mundial de Saúde, Genebra, Suíça.

\section{Resultados}

O estudo prospectivo para avaliação pré-natal da maturidade fetal foi realizado em 121 gestantes de alto risco internadas entre janeiro de 1990 e janeiro de 1995 no Hospital São Paulo Universidade Federal de São Paulo.

$\mathrm{O}$ diabetes mellitus (48 casos) e as síndromes hipertensivas (41 pacientes) foram as patologias preponderantes, representando $73 \%$ do total. A isoimunização pelo fator $\mathrm{Rh}$, presente em 14 grávidas, foi a terceira principal indicação para o estudo da maturidade. Diferentes patologias maternas e fetais (18 casos) completaram a casuística das 121 gestações de alto risco analisadas neste estudo (Tabela 1).

Tabela 1 - Patologias intercorrentes em 121 gestações de alto risco submetidas à avaliação pré-natal da maturidade fetal.

\begin{tabular}{lcc} 
PATOLOGIA & NÚMERO & PORCENTAGEM \\
\hline Diabetes mellitus & 48 & 39,6 \\
Sindromes hipertensivas & 41 & 33,9 \\
Isoimunização Rh & 14 & 11,6 \\
Malformações fetais & 06 & 4,9 \\
Polidrâmnio & 2 & 1,6 \\
Nefropatia materna & 2 & 1,6 \\
Trabalho de parto prematuro & 2 & 1,6 \\
Lúpus eritematoso sistêmico & 2 & 1,6 \\
Anemia falciforme & 1 & 0,8 \\
Tuberculose & 1 & 0,8 \\
Cardiopatia & 1 & 0,8 \\
Epilepsia & 1 & 0,8 \\
TOTAL & 121 & 100.0 \\
\hline
\end{tabular}

A Tabela 2 apresenta as características maternas (idade e número e porcentagem de cesarianas) e perinatais (idade gestacional e peso de nascimento, porcentagem de SDR, PIG, GIG e óbitos neonatais) das 121 gestações analisadas (grupo total), assim como em gestações complicadas por diabetes mellitus (DM) e por síndromes hipertensivas (SH).
Tabela 2 - Características maternas e perinatais de 121 gestações de alto risco submetidas à avaliação pré-natal da maturidade fetal. Os dados referentes às pacientes com diabetes mellitus e sindromes hipertensivas são apresentados separadamente.

\begin{tabular}{lccc}
\hline & DM [48] & SH [41] & Total [121] \\
\hline Idade em anos* & $32.1( \pm 7.1)$ & $29.0( \pm 7.0)$ & $30.1( \pm 6.8)$ \\
Cesárea $(\%) \S$ & $40(83.3)$ & $29(70.7)$ & $91(75.2)$ \\
Peso $(\mathrm{g}) *$ & $3116.8( \pm 782.6)$ & $2280.2( \pm 588.4)$ & $2646.3( \pm 801.6)$ \\
IG $($ sem)* & $36.7( \pm 1.8)$ & $35.8( \pm 2.3)$ & $36.0( \pm 2.4)$ \\
PIG $(\%) \S$ & $1(2.0)$ & $11(26.8)$ & $17(14.0)$ \\
GIG $(\%) \S$ & $1(29.1)$ & $2(4.9)$ & $18(4.9)$ \\
DR $(\%) \S$ & $12(25.0)$ & $9(21.9)$ & $33(27.2)$ \\
Óbito Perinatal $(\%) \S$ & $3(6.2)$ & $4(9.7)$ & $12(9.9)$ \\
& & & \\
\hline
\end{tabular}

* - Média e desvio padrão

$\S$ - Porcentagem

DM - Diabetes mellitus

$\mathrm{SH}$ - Síndromes hipertensivas

A Figura 1 apresenta a curva "ROC" para os valores da relação $\mathrm{L} / \mathrm{E}$ relativos à sensibilidade $\mathrm{e}$ especificidade obtidas em diferentes pontos de corte. Verificou-se que o ponto de corte em que houve o maior equilíbrio entre as duas características foi 1,4 (sensibilidade de $72 \%$ e especificidade de $81 \%$ ). O outro ponto de corte mais próximo do contrabalanço ideal foi a relação $\mathrm{L} / \mathrm{E}$ igual a 1,5, este, porém, com maior perda de especificidade (sensibilidade de $75 \%$ e especificidade de $72 \%$ ).

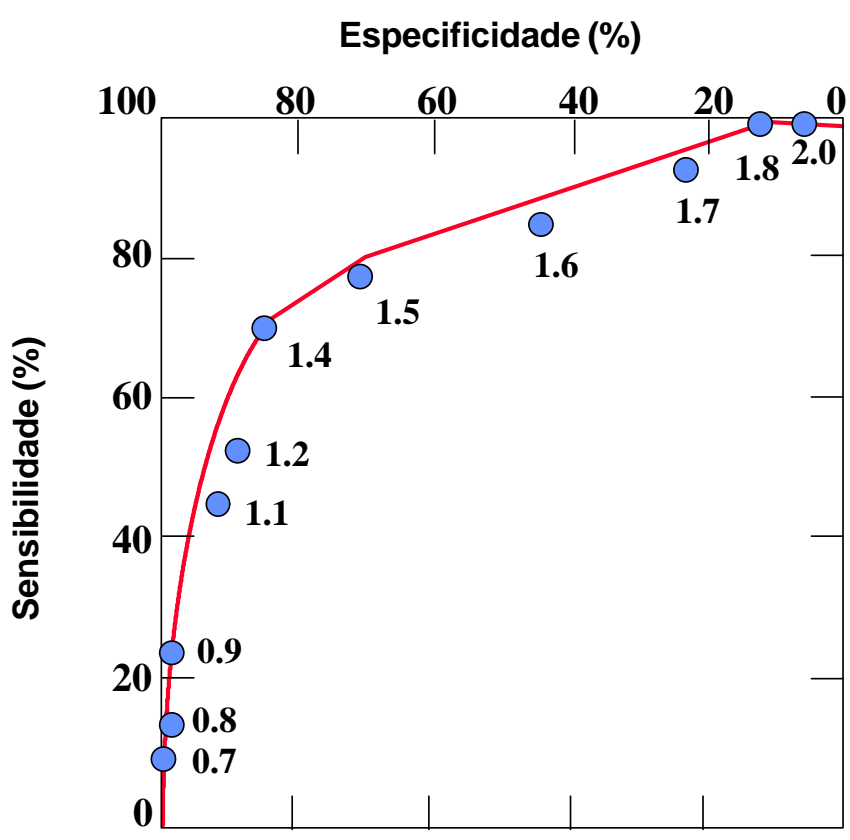

Figura 1 - Curva "ROC" para avaliação da acurácia de diferentes pontos de corte da relação L/E como um teste diagnóstico para ocorrência de desconforto respiratório neonatal em 121 gestações de alto risco. 
Para a adequada comparação do desempenho dos diferentes testes apresentamos, na Figura 2, a sensibilidade e a especificidade de cada um deles nas 121 gestações de alto risco estudadas. Constatou-se que o perfil pulmonar e teste de Clements obtiveram a maior sensibilidade entre os quatro testes e que a presença de PG foi aquele que apresentou a melhor especificidade. Embora a relação $\mathrm{L} / \mathrm{E}$ tenha demonstrado um desempenho equilibrado, perdeu em sensibilidade quando comparada ao teste de Clements e ao perfil pulmonar, e em especificidade quando comparada à presença de $\mathrm{PG}$.

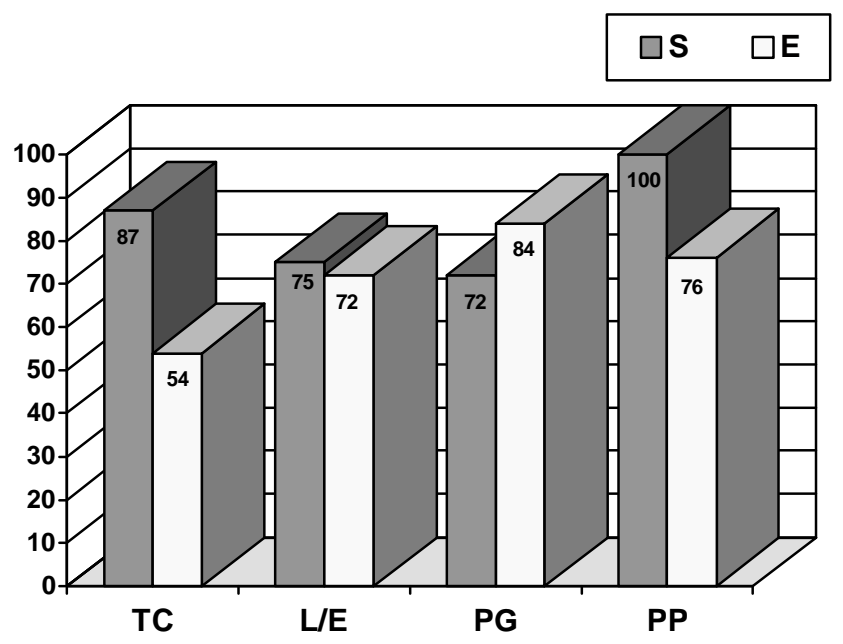

Figura 2 - Sensibilidade (S) e Especificidade (E) do teste de Clements (TC), relação L/E (L/E), presença de fosfatidilglicerol (PG) e perfil pulmonar (PP) em 121 gestações de alto risco.

\section{Discussão}

O estudo em gestações de alto risco demonstrou que o diabetes mellitus e as síndromes hipertensivas foram as principais indicações para a avaliação da maturidade pulmonar fetal em um período de cinco anos no Hospital São Paulo. Isoimunização pelo fator Rh, malformações fetais, nefropatia materna, lúpus eritematoso sistêmico e trabalho de parto prematuro foram outras indicações, que refletiram o cotidiano de um centro hospitalar terciário no Brasil, para o qual são encaminhadas gestantes de alto risco de diversas unidades básicas de saúde.

A maior porcentagem de gestantes diabéticas e hipertensas também pode ser explicada pela alta prevalência destas patologias na população geral em idade reprodutiva. O diabetes mellitus apresenta
A Figura 3 demonstra os valores preditivos positivo e negativo do teste de Clements, da relação $\mathrm{L} / \mathrm{E}$, da presença de PG e do perfil pulmonar. Verificou-se que todos os testes apresentaram elevados valores preditivos negativos, revelando alta probabilidade de ausência de desconforto respiratório quando o resultado é negativo ("maduro"). Os valores preditivos positivos, todavia, foram baixos para todos os testes, particularmente para o teste de Clements e para a relação L/E, sendo menos eficientes em avaliar a probabilidade de ocorrência de SDR frente a um resultado positivo ("imaturo").

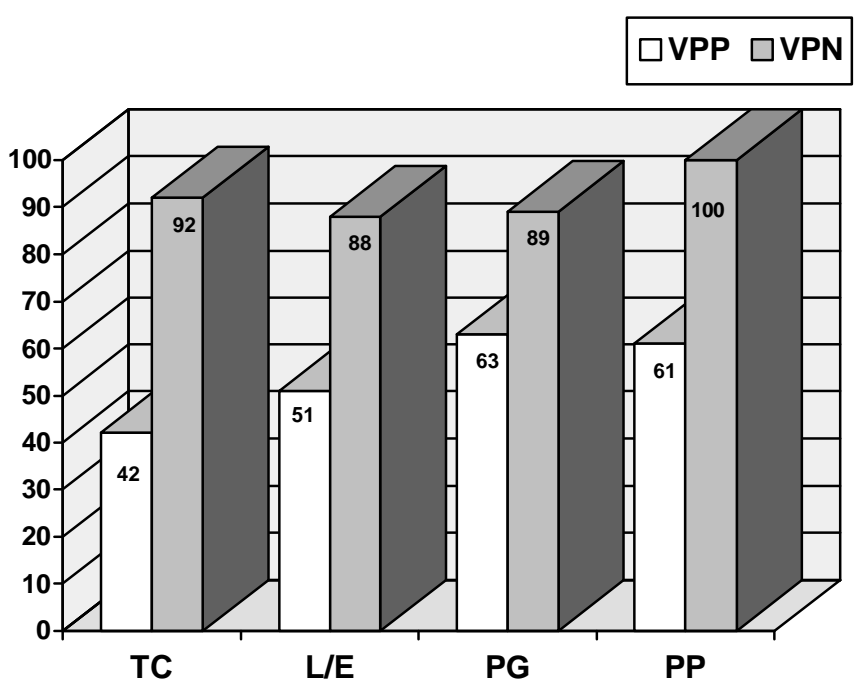

Figura 3 - Valores preditivos positivo (VPP) e negativo (VPN) do teste de Clements (TC), relação $L / E(L / E)$, presença de fosfatidilglicerol (PG) e perfil pulmonar (PP) em relação à ocorrência de DR em 121 gestações de alto risco.

incidência variável de $1.5 \%$ a $35 \%$ na gravidez, dependendo da população analisada ${ }^{15}$. Na Escola Paulista de Medicina (UNIFESP), a prevalência de diabete na população obstétrica geral foi de 4,3\% entre 1980 e 1985. A implantação de um programa de rastreamento sistemático do diabetes mellitus no pré-natal elevou a prevalência para $8 \%$ a $10 \%$ na mesma instituição, indicando que a ocorrência "espontânea" era subestimada ${ }^{3}$. As sindromes hipertensivas na gestação foram a segunda principal indicação para avaliação da maturidade fetal. A hipertensão na gravidez tem incidência variável de $5 \%$ a $10 \%$ e configura um contingente especial de pacientes que merece assistência médica individualizada, já que é uma das principais causas de morte materna no Brasil e em muitas regiões do mundo ${ }^{7}$. Além disso, a hipertensão arterial determina importante risco perinatal em função da freqüente associação com prematuridade 
e graus variáveis de asfixia. Nos Estados Unidos, onde as taxas de prematuridade geral variam de $4.9 \%$ a $10 \%$, evidenciou-se que cerca de $5,5 \%$ a $13 \%$ de todos os prematuros foram associados à pré-eclâmpsia. Em casos de pré-eclâmpsia grave e eclâmpsia as cifras de prematuridade oscilaram entre 59\% e 80\% ${ }^{1}$. Na Escola Paulista de Medicina (UNIFESP) verificou-se que, em cerca de $70 \%$ dos casos de pré-eclâmpsia sobreposta à hipertensão crônica e de eclâmpsia, o parto foi realizado no período pré-termo, geralmente por indicação materna, culminando com excessiva morbidade respiratória neonatal ${ }^{18}$.

Em relação ao desempenho dos testes para predizer a ocorrência de SDR, verificou-se que o perfil pulmonar e o teste de Clements apresentaram maior sensibilidade do que a relação L/E e a presença de fosfatidilglicerol. A sensibilidade constituiu o parâmetro de maior importância clínica neste estudo, já que identificou os recémnascidos imaturos que desenvolveram SDR. É exatamente este grupo de conceptos que poderia beneficiar-se com a maior permanência intra-útero ou por meio da utilização materna de corticosteróides destinada a acelerar a maturação pulmonar.

Por outro lado, a presença de $\mathrm{PG}$ e a relação $\mathrm{L} / \mathrm{E}$ apresentaram maior especificidade do que o teste de Clements. A especificidade identificou os conceptos "maduros", favorecendo a conduta ativa em gestações de risco. A baixa especificidade determinou valor preditivo positivo igualmente baixo. Este erro apresenta, porém, menor repercussão perinatal do que estimar a ausência de doença em neonatos que venham a ser retirados do útero e desenvolvam SDR no berçário (valor preditivo negativo). O VPN do teste de Clements foi de $92 \%$, resultado altamente expressivo na identificação dos fetos "maduros". Esse resultado foi similar ao de Schlueter et al $^{16}$, que verificaram VPN de 99,7\% para o teste de Clements em 205 gestações submetidas à amniocentese até 24 horas antes do parto. Taso e Zachman ${ }^{19}$ também apuraram VPN de 99\% em 690 testes, nos quais o líquido amniótico havia sido obtido até 72 horas antes do nascimento. Desta forma, acreditamos que o teste de Clements deva preceder qualquer outra prova para maturidade pulmonar, funcionando como rastreamento inicial. Frente à formação do anel de bolhas até o terceiro tubo, a maturidade estará praticamente garantida, com poucas chances de erro. As técnicas cromatográficas poderiam, portanto, ser reservadas para os casos de ausência de "positividade" no terceiro tubo e urgência para a decisão obstétrica.

A presença de $P G$ foi o indicador de maturidade pulmonar com a mais alta especificidade dentre todos os testes. Este resultado demonstra a segurança da prova em identificar os conceptos "maduros" que não desenvolverão SDR, mesmo em gestações de alto risco, confirmando as observações iniciais de Kulovich e Gluck ${ }^{13}$ e Golde e Mosley ${ }^{10}$.

O teste de maior acurácia global foi o perfil pulmonar sem nenhum falso positivo e com especificidade de $76 \%$. Este resultado ratifica a literatura internacional que revela que a somatória dos testes atinge índices mais altos de preditividade do que qualquer um utilizado isoladamente. De outra parte, a análise de parâmetros cromatográficos simultâneos não é necessariamente mais complexa ou mais demorada do que um teste isolado (exceto para o teste de Clements), apresentando vantagens para a melhor identificação de conceptos "maduros" e "imaturos" além da possibilidade de classificação quanto a categorias de risco. O perfil pulmonar também foi o teste com maior valor preditivo negativo $(100 \%)$ em gestações complicadas por diabetes mellitus e por síndromes hipertensivas. A especificidade, entretanto, foi relativamente baixa, não só para o perfil pulmonar, mas também para as outras provas.

Finalmente, cumpre salientar que sempre que o teste empregado apresentar baixa especificidade em gestações de alto risco, existe a possibilidade de haver incremento da mortalidade neonatal por prematuridade sem que haja, necessariamente, correspondente redução das perdas fetais.

\section{SUMMARY}

The objective was to evaluate the accuracy of the foam stability test, lecithin/sphingomyelin $(L S)$ ratio, presence of phosphatidylglycerol $(P G)$ and lung profile $(L / S$ ratio $>1.7$ and $P G$ present simultaneously) in 121 consecutive high-risk gestations at the São Paulo Hospital from January 1990 to January 1995. Delivery occurred within 3 days of fetal lung maturation testing. This is a prospective study in which the sensitivity, specificity, positive (PPV) and negative predictive value $(N P V)$ of all the tests were determined. Neonatal respiratory outcome and amniocentesis results were stratified by gestational age for comparison. The distribution of the studied population according to maternal pathology was diabetes mellitus (48), hypertensive disorders (41), $R h$ isoimmunization (14) and miscellaneous (18). Respiratory distress $(R D)$ was present in 33 infants (27.2\%), mainly in the diabetic group. There was no false negative using lung profile (all patients) and foam stability tests among hypertensive pregnancies (specificity 100\%), but there were about $20 \%$ to $50 \%$ false positives in the other tests. Overall, all four tests had a low PPV: $23 \%$ for foam test, $51 \%$ for L/S ratio, $63 \%$ 
for $P G, 61 \%$ for lung profile, and high NPV: $92 \%$ for foam test, $88 \%$ for $L / S$ ratio, $89 \%$ for $P G$ and $100 \%$ for lung profile. All tests had less accuracy in the diabetic pregnant women. This study shows that the presence of $P G$ and $L / S$ ratio $>1.7$ in the amniotic fluid of high-risk pregnancies confirms maturity with a very low risk to develop $R D$ and that the foam stability test was useful as a first-line test to predict the absence of surfactant-deficient respiratory distress syndrome, particularly in hypertensive pregnant women.

KEY WORDS: Fetal lung maturation. Amniocentesis. Arterial hypertension. Diabetes. Clements test. L/S ratio.

\section{Referências}

1. American College of Obstetrician and Gynecologists (US) Preterm labor. Technical Bulletin n. 133. October 1989.

2. Bertini AM, Camano L. Diagnóstico e tratamento prénatal da maturidade pulmonar. In: Kopelman BI, editor. Distúrbios respiratórios do recém-nascido. São Paulo: Atheneu; 1984. p. 97-111.

3. Bertini AM, Camano L. Morbidade e mortalidade perinatais no binômio diabetes mellitus e gravidez. Rev Bras Ginecol Obstet 1992;14: 183-8.

4. Caspi E, Schreyer I, Schereyer P, Weinraub Z, Tamir I. Amniotic fluid volume, total phospholipids concentration and L/S ratio in term pregnancies. Obstet Gynecol 1975; 46:684-7.

5. Clements JA, Platzker AC, Tierney DF, Hobel CJ, Creasy RK, Margolis AJ, et al. Assessment of the risk of the respiratory distress syndrome by a rapid test for surfactant in amniotic fluid. $\mathrm{N}$ Engl J Med 1972; 286:1077-81.

6. Creasy RK. Preterm labor. In: Eden RD, Boehm F, editors. Assessment and care of the fetus: physiological, clinical and medicolegal principles. Norwalk, Connecticut: Appleton-Lange; 1990. p. 617-30.

7. El-Kadre D, Deláscio D. Hipertensão na gravidez. $3^{\text {rd }}$ ed. São Paulo: Sarvier; 1991. 175 p.

8. Garite TJ, Freeman RK, Nageotte MP. Fetal maturity cascade: a rapid and cost-effective method for fetal lung maturity testing. Obstet Gynecol 1986; 67: 619-22.
9. Gluck L, Kulovich MV, Borer RC Jr, Brenner PH, Anderson GG, Spellacy WN. Diagnosis of the respiratory distress syndrome by amniocentesis. Am J Obstet Gynecol 1971; 109: 440-5.

10. Golde SH, Mosley GH. A blind comparison study of the lung phospholipid profile, fluorescence microviscosimetry, and the lecithin/ sphingomyelin ratio. Am J Obstet Gynecol 1980; 136: $222-7$.

11. Gotelli GR, Stanfill RE, Kabra PM, Farina FA, Marton LJ. Simultaneous determination of phosphatidylglycerol and the lecithin/ sphingomyelin ratio in amniotic fluid. Clin Chem 1978; 24: 1144-6.

12. Hallman M, Kulovich M, Kirkpatrick E, Surgarman RG, Gluck L. Phosphatidylinositol and phosphatidylglycerol in amniotic fluid: indices of lung maturity. Am J Obstet Gynecol 1976; 125: 613-7.

13. Kulovich MV, Gluck L. The lung profile. II. Complicated pregnancy. Am J Obstet Gynecol 1979; 135: 64-70.

14. Lowry MF. The problems of being born too soon. In: Studd J, editor. Progress in obstetrics e gynecology. Edinburgh: Churchill Livingstone; 1989. p.161-74.

15. Pedersen J, Bojsen-Mller B, Poulsen H. Blood sugar in newborn infants of diabetic mothers. Acta Endocrinol 1954; 15: 33-52.

16. Schlueter MA, Phibbs RH, Creasy RK, Clements JA, Tooley WH. Antenatal prediction of graduated risk of hyaline membrane disease by amniotic fluid foam test for surfactant. Am J Obstet Gynecol 1979; 134: 761-7.

17. Stubblefield P. Causes and prevention of preterm labor: An overview. In: Fuchs F, Stubblefield PG, editors. Preterm birth: causes, prevention and management. New York: MacMillan; 1984. p.3-20.

18. Taborda WC, El-Kadre D, Sassi N, Bertini AM, Camano L. Perinatal morbidity and mortality of the hypertensive disorders in pregnancy. Clin Exp Hypert 1991; B10:262/abstracts.

19. Taso FH, Zachman RD. Prenatal assessment of fetal lung maturation: a critical review of amniotic fluid phospholipid tests. In: Farrel PM, editor. Lung development: biological and clinical perspectives. Academic Press: New York; 1982, v.2, p.167-203. 